\title{
An umbilical venous catheter complication presented as acute abdomen. Case report
}

\author{
Assistant Professor Mustafa O. Oztan, M.D. ${ }^{a}$, Ozkan Ilhan, M.D. ${ }^{b}$, Elif Abay, M.D. ${ }^{c}$ and \\ Professor Gokhan Koyluoglu, M.D. ${ }^{a}$
}

\begin{abstract}
Umbilical venous catheterization has become a widely accepted intravenous route for premature babies. These catheters allow administration of parenteral nutrition and medication and facilitate blood sampling. Besides these benefits, they also have significant potential complications like portal vein thrombosis, infection, vascular or hepatic injury, arrhythmia and sepsis. One of the rare but important complication is extravasation of the fluids due to misplacement of the catheter. The typical symptoms of this condition are sudden deterioration, hepatic enlargement, hematocrit drop, hypotension and abdominal distension. We herein present a premature newborn with unusual acute abdomen findings suggesting a surgical pathology after the extravasation of total parenteral nutrition into the abdomen.

Key words: umbilical veins, catheterization, newborn, extravasation of parenteral nutrition.
\end{abstract}

http:/ /dx.doi.org/10.5546/aap.2016.eng.e429

\section{INTRODUCTION}

Umbilical vein catheterisation is a common procedure for vascular access at preterm newborns. ${ }^{1}$ It is used for intravenous fluid and drug administration, parenteral nutrition and exchange transfusion. ${ }^{2}$ This procedure in neonates is not always uneventful. The complications include malposition, portal vein thrombosis, hepatic necrosis, arrhythmia, pericardial perforation/tamponade, hydrothorax and systemic complications like sepsis and thromboembolies. ${ }^{3,4}$ Even more there is the possibility of extravasation of the fluid into the

a. Department of Pediatric Surgery, Katip Celebi University Faculty of Medicine. Izmir, Turkey.

b. Department of Pediatrics, Division of Neonatology, Tepecik Research and Training Hospital. Izmir, Turkey.

c. Department of Pediatric Surgery, Tepecik Research and Training Hospital. Izmir, Turkey.

E-mail Address:

Mustafa O.Oztan, M.D.: mustafaonur.oztan@ikc.edu.tr

Funding: None.

Conflict of interest: None.

Received: 2-19-2016

Accepted: 6-21-2016 intraperitoneal space. The common reason of this complication is the misplacement of the catheter. We presented a newborn with ascites and hyponatremia after total parenteral nutrition administration via an umbilical venous catheter.

\section{Case Report}

A 24-week-gestational age, $630 \mathrm{~g}$ female newborn was born with Apgar scores of 4 and 6 at one and five minutes, respectively. The baby was depressed shortly after birth and presented with bradycardia and desaturation. She was intubated after delivery and given surfactant for respiratory distress. A 3.5 Fr-UVC was inserted for delivering of total parenteral nutrition (TPN) on day one with the tip located at the level of T10 on radiographs, superimposed over the liver (Figure 1). TPN was started by continuous infusion through the UVC. Separate intravenous lines were used for blood samples and medication. On day 5 of life, abdominal distension and abdominal erythema developed, and the respiratory distress worsened. The radiography revealed a gasless abdomen and abdomen ultrasonography revealed significant amount of free fluid but no pathology at the liver. The laboratory tests showed hyponatremia $(127 \mathrm{mmol} / \mathrm{L})$ and thrombocytopenia; arterial blood gas, hematocrit, liver enzymes, blood sugar and $C$ - reactive protein levels were within normal limits.

At first, a midgut volvulus and a rapid progressing necrotizing enterocolitis was suspected, because of the severe abdominal tenderness, hyperemia, distension on physical examination, gasless abdomen on x-ray and excess intraabdominal fluid on ultrasound examination. The upper gastrointestinal contrast study revealed no malrotation; midgut volvulus was not considered and a penrose drain was inserted for conservative management of suspected necrotizing enterocolitis or spontaneous intestinal perforation. After a right lower quadrant incision, approximately $80 \mathrm{ml}$ of milky-looking fluid (typical appearance and biochemical analysis was consistent with TPN) was drained. The abdominal distension resolved; a diagnosis of intraperitoneal 
extravasation of TPN from the UVC was made and the catheter was removed. The drain was removed at the following day because there was no discharge of fluid at the follow-up. The child was stable with no signs of sepsis or dehydration; she was treated with antibiotics and supportive fluid administration. The patient died because of sepsis on day 64 of life without association with this complication.

\section{DISCUSSION}

Umbilical vein catheterisation is the most commonly used method of vascular access in neonatal intensive care. ${ }^{5}$ The advantages of this procedure are to facilitate blood sampling, TPN and drug administration, and to reduce stress and pain from repeated puncture of peripheral veins. ${ }^{6}$ The tip of the umbilical catheter must be placed over the diaphragm, at the junction of inferior

\section{Figure 1: Abdominal X-ray}

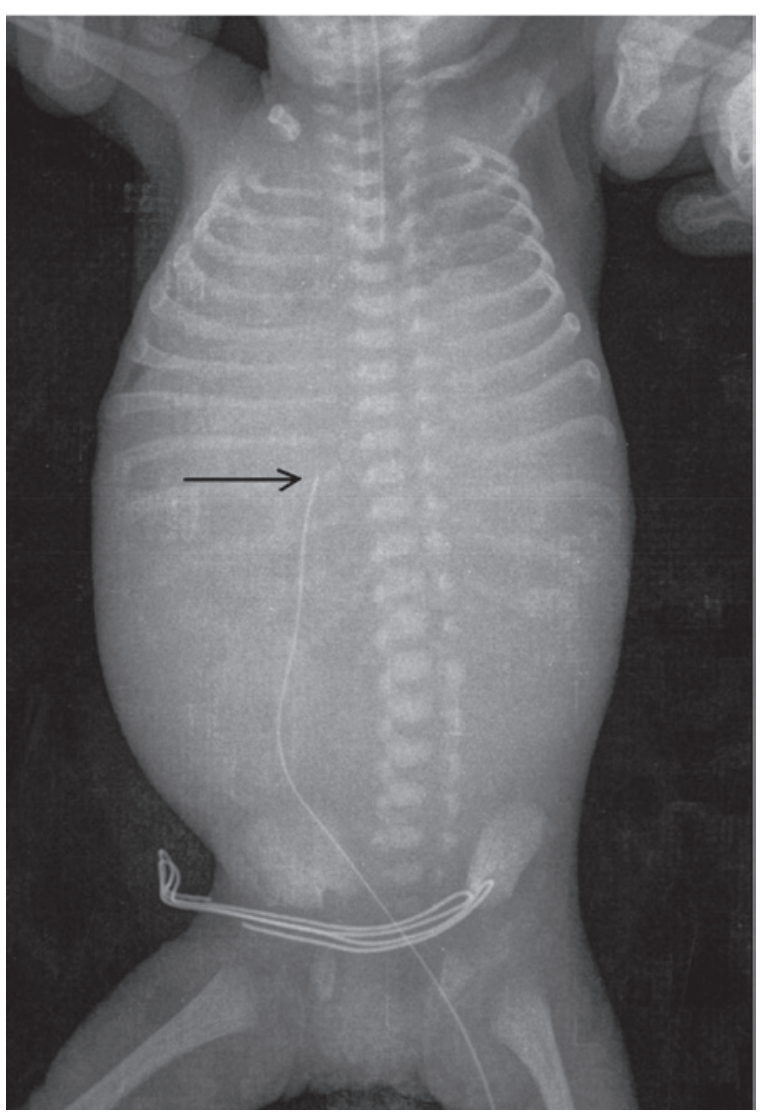

Abdominal X-ray of the patient at the $5^{\text {th }}$ day of umblical venous catheter placement. The tip of the catheter is at the level of the T10 vertebra (arrow). There is also distended gasless abdomen. vena cava and right atrium..$^{3,7}$

After catheterisation, the position of the catheter should be controlled with chest and abdomen x-ray. The tip of the UVC must be found between the $8^{\text {th }}$ and $9^{\text {th }}$ thoracic vertebrae or just above the diaphragm at the x-ray. ${ }^{8}$ In the article of Greenberg et al., the position UVC tips were compared with $x$-ray and ultrasound in 79 patients. ${ }^{9}$ It has been found that two thirds of catheters below $10^{\text {th }}$ vertebrae on $\mathrm{x}$-rays were found at the liver level at the ultrasound. In such cases the radiologist has an important role to alert the pediatrician. ${ }^{1}$ In our case, the tip of the UVC was located at T10 on radiograph and this situation has not been identified as inadequate by the physicians, therefore, nobody modified the catheter before to start with the parenteral nutrition.

An UVC can remain in place for up to 14 days. ${ }^{9}$ TPN should be delivered whenever possible through central lines such as percutaneous central line or UVC. TPN can also be administered by peripheral vein depending on the concentration of dextrose and osmolarity of the solution. If the concentration of dextrose is over $12.5 \%$ or the solution is hypertonic, the TPN should not be administered by peripheral vein. ${ }^{10}$

Many systemic but also local complications has been reported like hepatic laceration, hepatic collection, ascites, hepatic abscess formation, hepatic necrosis and vessel perforation after low placed UVCs. ${ }^{1,5,6,11}$ The length of using time of UVC may also related to the development of complications associated with the liver. ${ }^{11}$ The direct administration of TPN to liver may cause damage to the parenchyma of the liver and in time liver capsule get disrupted from this area causing free extravasation of TPN., ${ }^{1,12}$ The IVC also can cause direct laceration of the parenchyma by itself. $^{7}$

In our case, there was no change in hemoglobin concentration that indicated direct vessel perforation or no liver enzyme elevation that indicated hepatic damage. Abdominal ultrasonography showed also a normal liver tissue. Therefore we focused on the abdominal distention, which could be observed frequently in surgical pathologies. In this premature infant, midgut volvulus, spontaneous intestinal perforation and necrotizing enterocolitis were our first suspected etiologies.

Volvulus of the midgut occurs as a result of interruption of intestinal rotation and fixation. Small bowel and descending colon twist around 
the superior mesenteric artery resulting in vascular compromise leading to necrosis. We did an upper gastrointestinal contrast $X$-ray to visualize the duodenum and assess for malrotation and midgut volvulus. There was no duodenal obstruction or the classical "corkscrew" appearance of the misplaced duodenum.

Spontaneous intestinal perforation and necrotizing enterocolitis occur primarily in premature infants. Systemic signs are nonspecific and include apnea, respiratory failure, lethargy, poor feeding, or temperature instability. Abdominal signs include distention, tenderness, or both, ileus or decreased bowel sounds, abdominal wall erythema, vomiting, and bilious drainage. The treatment options are variable depending to the condition of the patient. Because our patient was an extremely low birth weight premature and had thrombocytopenia, the only option to diagnose and also treat necrotizing enterocolitis or spontaneous intestinal perforation was to insert a penrose drain and wait. During this intervention we saw that the discharge was consisted with TPN which we did not expect, as all the laboratory and abdominal ultrasonography were normal.

Coley et al. ${ }^{1}$ reported that catheter tips were at or below T10 at initial placement in seven of eight patients with liver erosion and TPN ascites by UVCs. As in our patient, the position of the UVC tip must be corrected but also interpreted correctly with X-ray after insertion, and must be removed or the position must be modified in case of malposition. We considered in this case that malposition of the UVC tip could cause TPN ascites as Coley et al. stated.

In conclusion, there are so many complications related with the usage of umbilical catheters; some are relatively minor and some are potentially life-treating. ${ }^{11}$ It is essential to remember that unexpected clinical situations may be complications of misplacement of UVC. Like in our case, although abdominal ultrasonography showed no pathology at the liver. In patients with acute abdominal distension, extravasation of TPN must be considered as a possible etiology in addition to surgical pathologies.

\section{REFERENCES}

1. Coley BD, Seguin J, Cordero L, Hogan MJ, et al. Neonatal total parenteral nutrition ascites from liver erosion by umbilical vein catheters. Pediatr Radiol 1998;28(12):923-7.

2. Hui JY, Lo KK, Lo J, Chan ML, et al. Neonatal total parenteral nutrition ascites secondary to umbilical venous catheterisation. J HK Coll Radiol 2001;4:288-90.

3. Nash P. Umbilical catheters, placement, and complication management. J Infus Nurs 2006;29(6):346-52.

4. Mohan MS, Patole SK. Neonatal ascites and hyponatremia following umbilical venous catheterization. J Paediatr Child Health 2002;38(6):612-4.

5. Yigiter M,Arda IS, Hicsonmez A. Hepaticlaceration because of malpositioning of the umbilical vein catheter: case report and literature review. J Pediatr Surg 2008;43(5):E39-41.

6. Bothur-Nowacka J, Czech-Kowalska J, Gruszfeld D, Nowakowska-Rysz M, et al. Complications of umbilical vein catherisation. Case report. Pol J Radiol 2011;76(3):70-3.

7. Egyepong J, Jain A, Chow P, Godambe S. Parenteral nutrition- ascites with acute renal failure as a complication from an umbilical venous catheter in an extremely low birth weight infant. BMJ Case Rep 2011;2011. pii: bcr0220113813.

8. Raisanen P,CasselI,Martin G, GrazianoK. Umbilical venous catheter complication in an infant with left-sided congenital diaphragmatic hernia: extravasation owing to hepatic vein catheterization. J Pediatr Surg 2010;45(12):e33-5.

9. Greenberg M, Movahad H, Peterson B, Bejar R. Placement of umbilical venous catheters with use of bedside real-time ultrasonography. J Pediatr 1995;126(4):633-5.

10. Chaudhari S, Kadam S. Total parenteral nutrition in neonates. Indian Pediatr 2006;43(11):953-64.

11. Gülcan H, Hanta D, Törer B, Temiz A, et al. Hepatic laceration as a life-threatening complication of umbilical venous catheterization. Turk J Pediatr 2011;53(3):342-5.

12. Hagerott HE, Kulkarni S, Restrepo R, Reeves-Garcia J. Clinical-radiologic features and treatment of hepatic lesions caused by inadvertent infusion of parenteral nutrition in liver parenchyma due to malposition of umbilical vein catheters. Pediatr Radiol 2014;44(7):810-5. 